\section{P-118 IMPROVING EXPERIENCE FOR RELATIVES OF DYING PATIENTS IN A CANCER CENTRE}

Martha Knowles, Richard Berman, Wendy Makin, Karen Anthony, Elizabeth Elliott. The Christie, Manchester, UK

\subsection{6/bmjspcare-2017-00133.117}

Background I felt strongly about improving the services for end of life relatives staying for long periods with their loved ones. I wanted to find out what they felt was missing from our care, and how we could improve their time with us.

Method I conducted a survey of twenty EOL relatives asking about their experience generally and what could be improved. I asked the questions in a safe place, questions around this are obviously of a sensitive nature and I wanted them to feel free to answer openly and honestly.

Results/findings

- Relatives want more information about what to expect during the dying process, such as physical signs and symptoms, as well as around what services are available.

- No facilities for relatives to make their own drinks and often feel they cannot approach busy staff.

- No facilities available for relatives who arrive without any of their own belongings.

Conclusion/actions

- I created A relative's guide for loved ones in the last days of life. This booklet contains information about signs and symptoms they may see in a dying loved one; services available across the hospital; and a summary about what support they offer.

- I instated a scheme where EOL relatives are entitled to a meal card activated with credit worth $£ 5$ per day so they are able to buy refreshments from the canteen and restaurant. This reactivates at midnight and can be used throughout their stay.

- I compiled a relative's overnight pack-a mini washbag, containing toothbrush, toothpaste, wipes and tissues for when they need to stay unexpectedly.

Good palliative care is essential - for a patient's loved ones as well as themselves. I would like to continue to make changes and create some positives in what can be otherwise a wholly negative experience.

\section{P-119 USE OF BACLOFEN IN A CONTINUOUS SUBCUTANEOUS INFUSION}

Helena Myles, Faith Cranfield. St. Francis Hospice, Dublin, Ireland

\subsection{6/bmjspcare-2017-00133.118}

Introduction This case report illustrates the effective use of a continuous subcutaneous infusion of baclofen to improve spasticity in a patient approaching end of life.

Report The patient was a 56 year old lady with a diagnosis of motor neurone disease (spinal onset; 'definite' by the El Escorial research criteria). She was admitted to the hospice for end of life care. She was severely dysarthric. She had a flicker of movement in her left hand, but no movement in her limbs otherwise. She communicated by nodding or shaking her head. She did not have a percutaneous endoscopic gastrostomy (PEG) tube in situ, and she was too frail for consideration of same. At the time of admission, she was taking baclofen $10 \mathrm{mg}$ po tds for spasticity.
As she weakened, she couldn't swallow her oral medications, necessitating discontinuation of her baclofen.

Three days later, she developed painful spasticity of her temporomandiibular joint muscles. She was very distressed by this symptom. It persisted over the following five days despite opioid analgesia and benzodiazepines. A literature review revealed a case report demonstrating the effective and well tolerated use of subcutaneous baclofen in a patient who could no longer take his medications via PEG.

A decision was made to commence a continuous subcutaneous infusion with bacofen $10 \mathrm{mg}$ over 24 hours and $\mathrm{NaCl}$ as the diluent. The intrathecal formulation of baclofen was used.

The following day, the patient reported improvement in her spasticity, and she was able to open her mouth. The improvement was sustained over the following four days, and she experienced no skin reactions at the infusion site. At this time she could no longer communicate, and she was beginning to die. The baclofen was discontinued as she was no longer benefitting.

Conclusion This case illustrates the effective and well-tolerated administration of baclofen via subcutaneous infusion. If appropriate, we may consider this route again.

\section{P-120 RETROSPECTIVE ANALYSIS OF THE TIMELINESS OF COMPLETION OF PAPERWORK AND COMMUNICATION TO GENERAL PRACTICE (GP) FOLLOWING DEATH}

Juliet Carmichael, Natasha Wiggins, Joanne Droney, Jayne Wood, Edith Obogagu. Royal Marsden NHS Trust Foundation Trust, London, UK

\subsection{6/bmjspcare-2017-00133.119}

Aims Primary aim: Assess compliance with national standards for completion of death certificates within 1 working day.

Secondary aim: Evaluate speed and form of communication of death to GP.

Background It is understood that effective and prompt communication to GPs facilitates provision of bereavement support to relatives after a death. Delayed paperwork delays funeral arrangements and causes unnecessary distress to those bereaved. There is very little research on how timing of paperwork and communication to the GP impacts on bereavement. This audit forms the initial work to address these issues in greater depth.

Methods Retrospective review of clinical notes in an acute cancer hospital between 1/12/15 and 31/3/16.

Results 87 patients died during the audit period. Death certificates were completed within 1 working day for $91.9 \%$ $(79 / 86)$ patients. 1 patient was referred for post mortem so was excluded here. GPs were informed of death via discharge summaries or telephone conversations. Overall it was documented that $78 \%(68 / 87)$ GPs were informed of the patient's death. Summaries were completed within 1 working day of death for $60.9 \%$ (53/87), 2 or more working days for $17.2 \%$ $(15 / 87)$. For $21.8 \%(19 / 87)$, the summary was not completed. $20 \%(17 / 87)$ deaths were communicated by phone.

Conclusion Overall, death certification was completed in a timely fashion. Discharge summaries were completed promptly for most, but $21.8 \%$ were never completed.

The discrepancy in communication with GPs highlights the need for interventions to ensure information is communicated promptly and effectively to facilitate ongoing bereavement support. This could be achieved with dedicated proformas, 
guidelines for staff, teaching sessions and the development of a standardised protocol for timely report to GP.

\section{P-121 SERVICE EVALUATION: WHAT HAPPENED TO HOSPICE IN-PATIENTS TRANSFERRED TO AN ACUTE HOSPITAL AND LESSONS LEARNED}

\author{
1,2Patricia Strubbe, ${ }^{2,3}$ Katrien Naessens. 'Sue Ryder Duchess of Kent Hospice, Reading, UK; \\ ${ }^{2}$ Sue Ryder, Reading, UK; ${ }^{3}$ Royal Berkshire Hospital Foundation Trust
}

10.1136/bmjspcare-2017-00133.120

The 2 hospice in-patient units combined have 27 in-patient beds. For every admission we document decisions about cardio-pulmonary resuscitation and transfer to acute hospital. Both units offer intra-venous treatments (blood products, bisphosphonates and antibiotics). Previously published audits ${ }^{1}$ were done in units were intravenous antibiotics were not available. This retrospective service evaluation was done in order to evaluate what happened to transferred patients and whether we can improve our practice in the future.

The authors looked at the clinical notes of all transfers to acute hospital between January 2014 and July 2015. Case finding relied on memory and documentation in admission books.

There were 16 transfer (involving 13 patients) 8 for diagnosis (fracture, MSCC, PE) 7 for treatment (electrolyte abnormality, neutropaenic sepsis and NIV initiation) and 1 for a post-surgical complication. Ten transfers happened during normal working hours and six out of usual working hours. Decisions tended to be well documented and consultants were involved in eleven cases.

In 11 instances the patient returned to the unit, 2 died in hospital, 2 were discharged home, 1 patient died 3 weeks later (location unclear) and in 12 cases the aim of the transfer was met.

Further analysis revealed that most transfer decisions were well documented. However what information was sent with the patient and criteria for return to the unit were not clear and patients lingered longer than intended in the acute hospital. We did not evaluate decisions not to transfer to hospital.

In future we aim

- Not only to document suitability for transfer on admission but also review this regularly.

- To document changes in clinical condition which may lead to transfer to acute hospital whether or not patient is transferred.

- On transfer to communicate doctor-to-doctor with clear goals and return criteria

- To liaise with hospital palliative care team.

\section{REFERENCES}

1. Castanheira T, Wright BA. Survey about patients transferred from a Specialist Palliative Care unit to an acute hospital setting . European Journal of Palliative Care 2014;21:219-221

2. Doidge $M$, Perkins P. Letter to the editor. European Journal of Palliative Care 2015;22(1)

\section{P-122 LIDOCAINE 5\% PATCH INITIATION AND ASSESSMENT}

Kirsty Lowe. Roxburghe House, Royal Victoria Hospital, Dundee, UK, DD2 1SP
Background The Scottish Palliative Care Guidelines (SPCG) provide guidance for initiating lidocaine $5 \%$ patches and assessing their efficacy. As most patients will respond within 1-2 weeks, patches should be reviewed around this time, by way of a patch-free trial, and discontinued if not beneficial.

In Roxburghe House hospice, Dundee, lidocaine patches are not routinely reviewed. My aim was to educate prescribers regarding national guidance, and to encourage them to review lidocaine patches 1-2 weeks after initiation.

Methods A record was kept of all inpatients who commenced lidocaine patches in Roxburghe between $1^{\text {st }}$ December 2015 and $29^{\text {th }}$ February 2016. Indication for starting patch, pain assessments and outcome of patch-free trial were all recorded.

Following cycle 1 I provided education sessions about the SPCG guidance for prescribers in Roxburghe. I introduced an assessment sheet based on the SPCG recommendations. I then repeated the data collection with patients who commenced lidocaine patches in Roxburghe House between $1^{\text {st }}$ July and $30^{\text {th }}$ September 2016.

Results Cycle 1 confirmed that the SPCG guidance was not being followed in Roxburghe House. Only one patient had a patch-free trial and the majority of patients had no pain assessment at 48 hours or 1-2 weeks.

In cycle $2100 \%$ of patients had pain scores documented, although only $50 \%$ had the new assessment form completed. Of the patients who survived past 1-2 weeks, $100 \%$ had clear instructions to their GP requesting review of the patch in the community.

Conclusions The introduction of a lidocaine patch assessment form has had some success so far in Roxburghe House, however further education is required to reinforce the importance of following the SPCG guidance. Other interventions which may be interesting would be providing education updates on the SPCG guidance for GPs and formulating an information leaflet for patients which provides instructions for how and when to initiate a patch-free trial.

\section{P-123 USING THE MODEL OF IMPROVEMENT TO INCREASE THE EFFICIENCY OF DISCHARGE MEDICATION PRESCRIBING IN PALLIATIVE CARE}

Rory Carrigan, Charles Daniels. St. Luke's Hospice (Harrow and Brent), Harrow, UK

\subsection{6/bmjspcare-2017-00133.122}

Aim To reduce the time taken and the quality of To Take Away (TTA) prescribing in a hospice setting

Background Using and learning from the model for improvement can help guide us through change. A hospice discharge process can be lengthy and complicated by the prescription of medications. It was hypothesised that too much time was being spent by clinicians on handwriting discharge prescriptions (FP10s). We proposed that typing, printing and electronically storing FP10 prescriptions would reduce the time taken and improve legibility.

Method An electronic TTA template was created in MS Word and stored on a secured network. We measured the time taken to produce and process handwritten and electronic TTAs after a period of change. The whole process was timed from creation to electronic submission to the pharmacy. Any enquiries or extra work sought by the pharmacy team was measured as an additional added time to this process. 